\title{
Detective Quantum Efficiency of the Medipix Pixel Detector
}

\author{
D. W. Davidson, J. Watt, L. Tlustos, B. Mikulec, M. Campbell, K. Mathieson, V. O’Shea, K. M. Smith, and \\ M. Rahman
}

\begin{abstract}
We have measured the intrinsic performance of a digital X-ray detector, the Medipix1, by examining the total detective quantum efficiency (DQE). We studied how the DQE depends on both the incident photon energy and spatial frequency. Reported here is the calculation of the detective quantum efficiency for the case of a $300 \mu \mathrm{m}$ thick silicon diode detector attached to the Medipix1 readout chip. This was done by determining the modulation transfer function and the noise power spectrum; together these allow the frequency component of the DQE to be calculated. $X$-ray absorption efficiency in the detector gives the dependence on incident energy. This system was found to have a DQE that peaked at 0.118 , using a dental $X$-ray source, and dropped to 0.049 at the Nyquist frequency of 2.94 line pairs per mm.
\end{abstract}

Index Terms-Detective quantum efficiency, image quality, Medipix, semiconductors.

\section{INTRODUCTION}

$\mathbf{T}$ O allow comparison between different imaging detectors a repeatable measurement that gives a good assessment of image quality is required. The detective quantum efficiency (DQE) is a measurement that should have an intrinsic value for a certain detection system. The DQE is defined as being the signal-to-noise ratio (SNR) transfer function and is given in [1] as

$$
\mathrm{DQE}=\frac{\mathrm{SNR}_{\mathrm{Out}}^{2}}{\mathrm{SNR}_{\mathrm{in}}^{2}}
$$

While the $\mathrm{SNR}_{\text {out }}$ can be calculated, by looking at the average and standard deviation in counts in a flat field acquisition, the $\mathrm{SNR}_{\mathrm{in}}$ is an immeasurable quantity. Therefore the calculation of the total DQE relies on examination of the following: the absorption efficiency of the detector material, for the spectrum of energies emitted by the source, the modulation transfer function (MTF) and the noise power spectrum (NPS). The first of these gives the energy dependent part of the DQE and the latter two give the spatial frequency dependence. The calculation of the total DQE comes as the product of these two factors.

Manuscript received December 3, 2002; revised May 8, 2003. The work of D. W. Davidson was supported by a PPARC CASE Award in association with the Rutherford Appleton Laboratories.

D. W. Davidson, J. Watt, K. Mathieson, V. O'Shea, K. M. Smith, and M. Rahman are with the Department of Physics and Astronomy, University of Glasgow, Glasgow G12 8 QQ, Scotland (e-mail: d.w.davidson@physics.gla.ac.uk).

L. Tlustos, B. Mikulec, and M. Campbell are with the EP Division, CERN, CH 1211 Geneva, Switzerland.

Digital Object Identifier 10.1109/TNS.2003.817382

\section{A. The Medipix Chip}

The Medipix readout used throughout this paper is based on the photon counting chip (PCC) designed at CERN with input from the Medipix collaboration. The working of the chip is explained in more detail in references [2] and [3]. The chip performs single photon counting in each pixel cell. Each pixel $(170 \mu \mathrm{m} \times 170 \mu \mathrm{m}$ in size $)$ is part of an array of 64 by 64 identical active cells. The array is bump-bonded to a semiconductor sensor of equal dimensions and segmentation. For this paper a $300 \mu \mathrm{m}$ thick silicon diode sensor medium was used.

The chip is read out to a PC through a custom made motherboard (Medipix-1 re-Usable Read Out System, MUROS-1, built at the National Institute for Nuclear Physics and High Energy Physics, NIKHEF, in Amsterdam). The software for running and setting up the chip operating parameters is provided by the University of Naples, Italy, and is based on the $\mathrm{C}$ environment within LabWindows.

In the near future the second generation of these chips will be available. These have two main advantages: the ability to be tiled to create a larger detector area than is currently available, and a reduced pixel size of $55 \mu \mathrm{m} \times 55 \mu \mathrm{m}$, which will improve spatial resolution. This chip is described in [4].

\section{ABSORPTION EFFICIENCY}

The absorption efficiency of the system depends on the source used and the detector medium. In this paper a $300 \mu \mathrm{m}$ silicon diode detector was used, bump bonded to the Medipix 1 readout chip. The source used for the measurements was a dental X-ray tube, the commercially available Planmeca Prostyle Intra X-ray Unit. The tube peak voltage was set to $60 \mathrm{kV}$ which results in a measured spectrum peaking at around $30 \mathrm{keV}$ and having a maximum energy of $60 \mathrm{keV}$ as shown in Fig. 1 [5].

The chip had an energy threshold set at $13 \mathrm{keV}$ so photons of energy below that were not registered by the system. Higher energy photons could be detected to some degree by the detector. The absorption efficiency at each energy can be calculated by looking at the absorption coefficient calculated for the material and thickness used. The data for such a calculation can be found in [6]. This provided the graph in Fig. 2 of detection efficiency for the energies used.

When the absorption efficiency is multiplied by the incident energy spectrum the detected energy spectrum is calculated and is shown in Fig. 3. As this is a photon counting device the different energies are weighted equally. This allows the absorption efficiency of the device to be calculated by looking at the integrals under the incident spectrum graph and the detected 


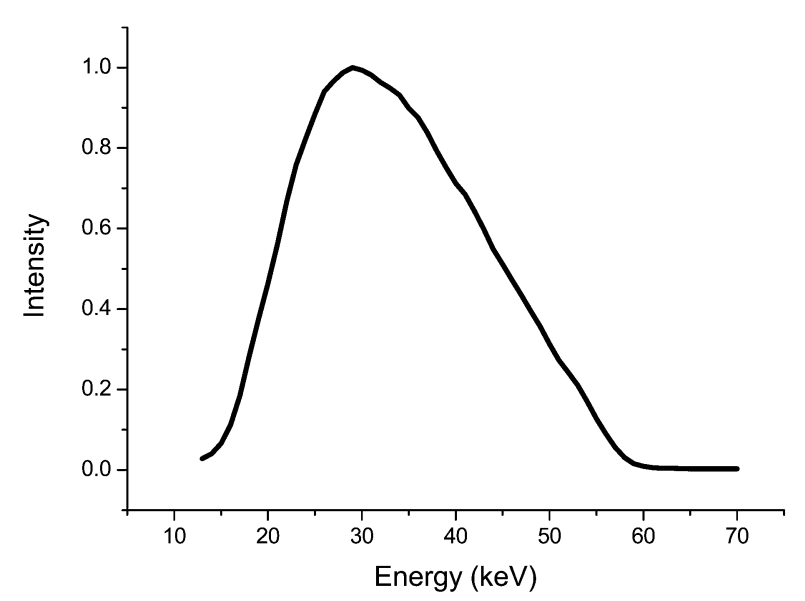

Fig. 1. Spectrum of X-ray source used (incident on device).

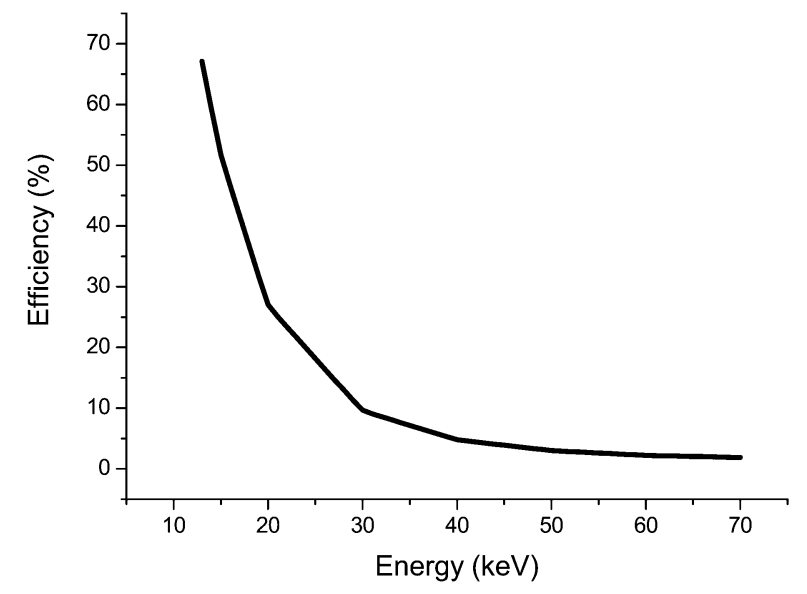

Fig. 2. Absorption Efficiency of $300 \mu \mathrm{m}$ of silicon at the energies present in the source.

spectrum graph. The detected spectrum has an integral of 0.112 times the integral of the incident spectrum graph therefore the device absorption efficiency is $11.2 \%$ for this setup.

\section{MOdULATION TRANSFER FUnCTION}

The modulation transfer function (MTF) gives the spatial response of a detector to a sinusoidal input stimulus. For the purposes of this paper the curve fitting line spread function measurement of the MTF is used. This technique is described in detail in [7] with only essential points recorded here. The response of a detector to an illuminated slit angled across the pixel plane gives a sampled line spread function (LSF). This LSF can be fitted by the function below, described in [7], formed by combining a Gaussian for the central part with an exponential for the tails

$\operatorname{LSF}(x)=a_{2} \exp \left[-\left\{\left(x-a_{1}\right) / a_{3}\right\}^{2} / 2\right]+a_{4} \exp \left[-\left|x-a_{1}\right| / a_{5}\right]$

where $a_{1}$ is the center of the fitted LSF, $a_{2}$ the weighting factor of the Gaussian function, $a_{3}$ the standard deviation of the

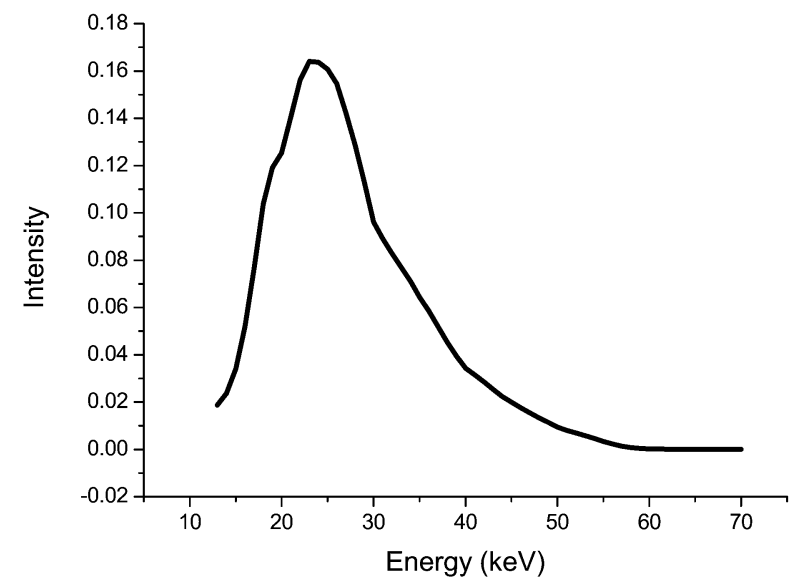

Fig. 3. Spectrum detected by the $300 \mu \mathrm{m}$ silicon detector medium. The intensity is to the same scale as the incident spectrum graph earlier.

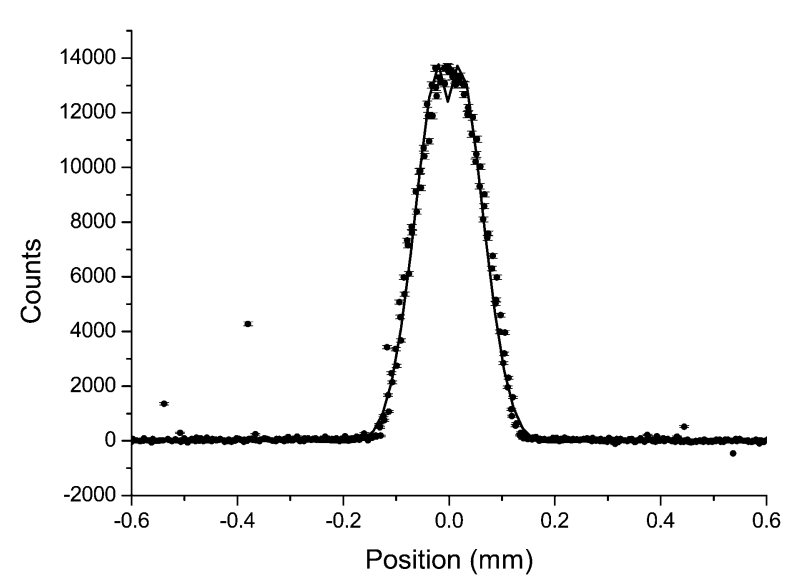

Fig. 4. Equation for a line spread function fitted, by least squares, to the counts data of the slit image. The coefficients as used for the MTF calculation were found to be: $20272,0.0547,-8245$, and 0.0432 for $a_{2}, a_{3}, a_{4}$, and $a_{5}$, respectively.

Gaussian function, $a_{4}$ the weighting factor of the exponential function and $a_{5}$ the slope of the exponential function.

In practice a slit of $40 \mu \mathrm{m}$ was placed in close contact with the detector. This was imaged by the dental source. The image was then flat field corrected to compensate for nonuniformities in the detector. A straight line was fitted to the imaged slit, using the least squares method, by taking the pixel with the highest counts in each column as the center of the slit and giving a weighting dependent on the number of counts in that pixel. The LSF, shown in Fig. 4, is the graph of the counts in a pixel against the distance from the center of the slit to the center of that pixel.

The MTF follows as the Fourier transform of the LSF. This can be done directly on the earlier equation to give, with the coefficients as fitted in Fig. 4

$\operatorname{MTF}(u)$

$$
=\frac{\sqrt{2 \pi} a_{2} a_{3} \exp \left[-2 \pi^{2} a_{3}^{2} u^{2}\right]+2 a_{4} a_{5} /\left(1+4 \pi^{2} a_{5}^{2} u^{2}\right)}{\sqrt{2 \pi} a_{2} a_{3}+2 a_{4} a_{5}} .
$$




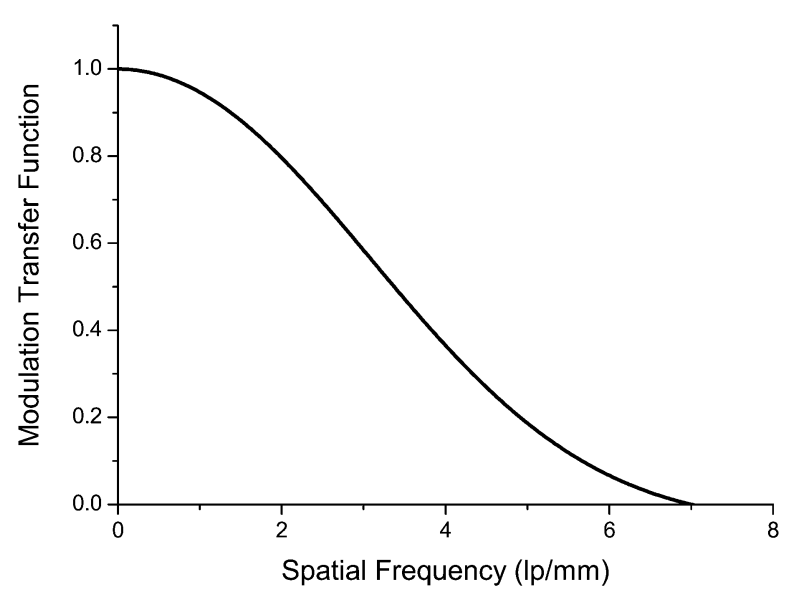

Fig. 5. Modulation transfer function of the detector using the coefficients from the line spread function fit.

The MTF shown in Fig. 5 was calculated in this manner. The standard comparison point on the MTF curve is the spatial frequency that has a MTF of 0.3 , here measured at 4.33 line pairs per millimeter $(\mathrm{lp} / \mathrm{mm})$. This is regarded as the maximum frequency able to be resolved by the detector. However for the calculation of the DQE only the data up to Nyquist frequency is used. The Nyquist frequency is given by the inverse of double the pixel pitch and is the limit at which the MTF has a physical meaning. The Nyquist frequency is the spatial frequency at which the LSF has two data points per line pair.

\section{NOISE POWER SPECTRUM}

The noise power spectrum (NPS) is a measure of the noise power per unit frequency, sometimes called the power spectral density. All images contain some noise, which, in a digital system, may be measured as the variance of the pixel to pixel fluctuations present in a flood image. The NPS provides a spectral decomposition of the variance to estimate the spatial frequency dependence of the noise.

The calculation of the NPS here is subject to a few assumptions. The system is assumed to have a linear response and be shift-invariant. For Medipix 1 the system is photon counting and linear across a large dynamic range (up to 32767 counts per pixel) and was always operated within this range. The requirement of shift-invariance (the output in response to a certain input is independent of position on the detector) is maintained providing the shift is by an integer number of pixels. This implies that here we are measuring the digital noise power spectrum.

The NPS is defined, in one dimension, as the Fourier transform of the noise autocorrelation function $\mathrm{C}_{a}(x)$. If $p(x)$ is the pixel value (for a digital system like Medipix1) of a one dimensional image at position $x, \tau$ is a dummy length variable and $L$ is the actual length of the data set then $\mathrm{C}_{a}(x)$ is defined in [8] as

$$
\mathrm{C}_{a}(x)=\lim _{L \rightarrow \infty} \frac{1}{L} \int_{-(L / 2)}^{L / 2} p(x+\tau) p^{*}(\tau) \mathrm{d} \tau
$$

where $p^{*}(x)$ would be the complex conjugate of $p(x)$. As $p(x)$ is real $p^{*}(x)=p(x)$. Completing the Fourier transform as calculated in [8] gives

$$
\operatorname{NPS}(u)=\lim _{L \rightarrow \infty} \frac{1}{L}|P(u)|^{2}
$$

where $P(u)$ is the Fourier transform of the data $p(x)$. This in turn leads to the expression used to calculate the NPS. In order to reduce low frequency fluctuations 100 images were taken, adjusted by an appropriate flat-field correction (as investigated in [9]) and ensemble averaged to give the expression below:

$$
\operatorname{NPS}(u)=\lim _{L \rightarrow \infty}\left\langle\frac{1}{L}\left|\int_{-(L / 2)}^{L / 2} p(x) \mathrm{e}^{-2 \pi i u x} \mathrm{~d} x\right|^{2}\right\rangle .
$$

However, for a digital (pixellated) system of pixel pitch $\Delta x, p(x)$ is evaluated at a set of discrete locations $n \Delta x$ where $n=0,1,2, \ldots, N$ and $L=N \Delta x$. This leads to the spatial frequency being sampled at discrete values $k \Delta u$ where $k=0, \pm 1, \pm 2, \ldots$ up to a maximum sample frequency called the Nyquist frequency which is given as

$$
u_{N}=\frac{1}{2 \Delta x} \text {. }
$$

Thus, the earlier equation is rewritten, for calculation from a discretely sampled source

$$
\operatorname{NPS}_{d}(u)=\lim _{L \rightarrow \infty}\left\langle\frac{\Delta x}{N}\left|\sum_{n=0}^{N-1} p(n \Delta x) \mathrm{e}^{-2 \pi i(k \Delta u)(n \Delta x)}\right|^{2}\right\rangle .
$$

In order to evaluate the NPS of the device for this paper 100 images of flood exposures were taken, using a dose of $170 \mu$ Grays each from the X-ray source. Each of these was adjusted with an appropriate flat field correction map to account for inhomogeneities in the detector medium, a standard imaging technique described in [9] and [10]. The average pixel value was then subtracted from all pixels in each image to leave a zero mean distribution. Only the inter pixel fluctuations remain. These data then had a "synthetic slit" applied to them by averaging down each column and recording in one row the values. The resultant one dimensional data from each image were fast Fourier transformed, giving 100 NPS distributions which were ensemble averaged to give the result in Fig. 6.

The synthetic slit was also applied in the opposite direction across the detector and found to give the same result. This was expected as the pixels are square, so the sampling frequency is the same in either direction, therefore, only one frequency direction is plotted here.

\section{Detective QuANTum EFFICIENCY}

The detective quantum efficiency is calculated using the results of the three previous sections. The energy component is simply the absorption efficiency and will provide a degree of limitation on the DQE. The frequency component was found by using the equation for the difference in the efficiency of the transfer of signal and noise through the detector found in [1]. This is given here as

$$
\operatorname{DQE}(f)=\frac{\operatorname{MTF}^{2}(f)}{\operatorname{NTF}^{2}(f)}
$$




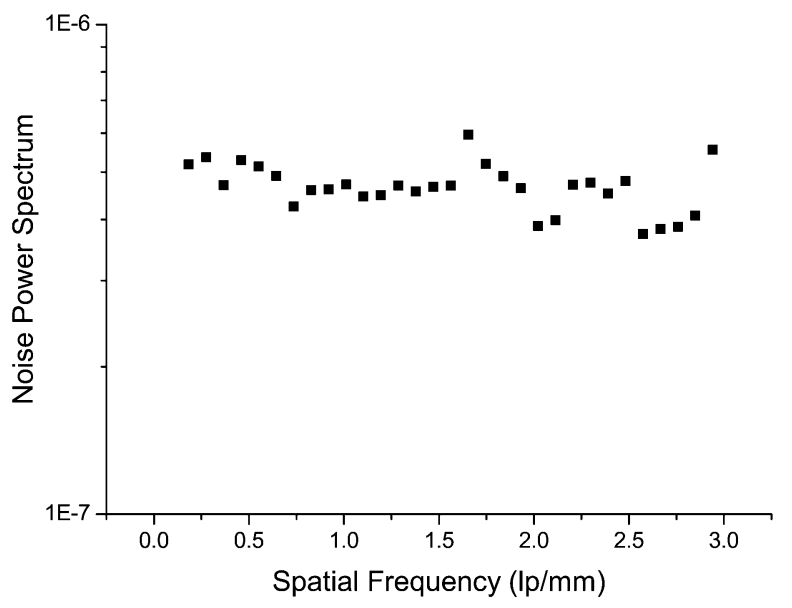

Fig. 6. The noise power spectrum of the Medipix 1 chip from flat-field images taken at $170 \mu \mathrm{Gy}$ dose. This was calculated using the synthetic slit approach on 100 images.

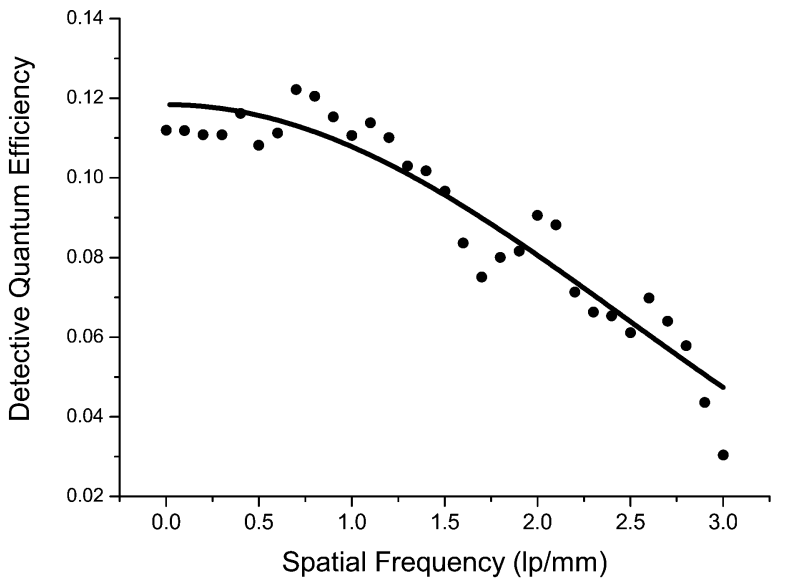

Fig. 7. The total detective quantum efficiency of the system against spatial frequency. The line is a least squares fit to the data points using the equation: $\mathrm{DQE}=\mathrm{A} \times \operatorname{sinc}^{2}(f l)$. The values for the maximum, $\mathrm{A}$, and pixel pitch, 1 , are found to be $0.1184 \pm 0.0010$ and $0.1678 \pm 0.0024$, respectively.

where the $\mathrm{NTF}^{2}(f)$ is the noise power spectrum normalized to one at zero frequency. This gives the equation of the total DQE as

$$
\mathrm{DQE}=\frac{\operatorname{MTF}^{2}(f) \times \mathrm{A}_{q}}{\operatorname{NTF}^{2}(f)}
$$

where $\mathrm{A}_{q}$ is the absorption coefficient of the system. This calculation results in the graph in Fig. 7 where the data points have been fitted by a $\left(\sin ^{2} x\right) / x^{2}$ function which allows the effective pixel pitch of the detector to be found. This effective pixel pitch is found to be $167.8 \mu \mathrm{m} \pm 2.4 \mu \mathrm{m}$ which agrees with the physical pixel size of $170 \mu \mathrm{m}$ within one standard deviation.

\section{DISCUSSION}

\section{A. Alternative Methods}

The detective quantum efficiency calculation recorded here is equivalent to the method of references [11] and [12]. The technique outlined in these references includes normalizing the NPS by the detected signal and therefore when the frequency dependent DQE is calculated this can be divided by the incident photon flux. This is in principle equivalent to multiplying the $\mathrm{DQE}(f)$ by the detected photon flux divided by the incident photon flux, which is the absorption efficiency of the detector.

\section{B. Possible Improvements}

The DQE is heavily influenced by the absorption efficiency and improving the system's DQE by any significant amount will primarily involve increasing this factor. The two options available are to increase the thickness of the detector or to use a higher $Z$ material. Using the same $\mathrm{X}$-ray source but with a $500 \mu \mathrm{m}$ silicon detector would increase the absorption efficiency to $17.4 \%$. Alternatively a $300 \mu \mathrm{m}$ thick gallium arsenide detector would give an absorption efficiency of $76.8 \%$. If the other factors could be kept the same then this change could give a DQE peaking at 0.768 .

Another way to improve the DQE slightly at the higher frequencies would be to try to hold the NPS flat (approximately) while decreasing the fall off of the MTF. This improvement will come with the introduction of the next generation of the readout chip, the Medipix2. The Medipix 2 chip will have a smaller pixel pitch of $55 \mu \mathrm{m}$ in each direction. If the effective pixel size of the fit was taken to be $55 \mu \mathrm{m}$, the DQE would be 0.109 at $2.94 \mathrm{lp} / \mathrm{mm}$ as apposed to 0.049 .

\section{CONCLUSION}

A measure of the intrinsic capabilities of the Medipix1 chip has been completed. The chip compares favorably with the commercially available Sens-A-Ray dental CCD chip. The reported peak DQE of the Sens-A-Ray is given as 0.030 using a $70 \mathrm{kV}$ peak X-ray tube [11]. The results obtained with the set up here give a DQE with a peak of 0.118 falling to 0.049 at the Nyquist frequency. However, with a detector optimized to the source used, and assuming a similar frequency dependence, a value perhaps as high as 0.768 could be achievable.

\section{ACKNOWLEDGMENT}

The authors would like to thank the Medipix Collaboration for designing and supplying the Medipix1 chip.

\section{REFERENCES}

[1] M. Sandborg, "Calculation and analysis of DQE for some image detectors in mammography,", ISSN 1102-1799, 1998.

[2] M. G. Bisogni et al., "Performance of a 4096 pixel counting chip,", CERN-EP/98-162, 1998

[3] M. Campbell et al., "Readout for a $64 \times 64$ pixel matrix with 15-bit single photon counting," IEEE Trans. Nucl. Sci., vol. 45, pp. 751-53, June 1998.

[4] X. Llopart et al., "Medipix2, a 64k pixel readout chip with $55 \mu \mathrm{m}$ square elements working in single photon counting mode" (in Proc. IEEE NSS/MIC 2001), IEEE Trans. Nucl. Sci., vol. 49, pp. 2279-2283, Oct. 2002. 
[5] C. Fröjdh and P. Nelvig, "Performance criteria for x-ray imaging sensors," Physica Medica, vol. XIV, pp. 10-12, 1997.

[6] Nat. Inst. Standards Technol.. (2002). [Online] http://physics.nist.gov/ PhysRefData/XrayMassCoef/ElemTab/z14.html

[7] F. Yin, M. L. Giger, and K. Doi, "Measurement of the presampling modulation transfer function of film digitizers using a curve fitting technique," Med. Phys., vol. 17, no. 6, pp. 962-66, 1990.

[8] M. B. Williams, P. A. Mangiafico, and P. U. Simoni, "Noise power spectra of images from digital mammography detectors," Med. Phys., vol. 26, no. 7, pp. 1279-93, 1999.
[9] D. W. Davidson et al., "Limitations to flat-field correction methods when using an X-ray spectrum" (in Proc. IWoRiD 2002), in Nucl. Instrum. Methods A, vol. 509, 2003, pp. 146-150.

[10] B. Mikulec, "Single photon detection with semiconductor pixel arrays for medical imaging applications,", CERN-thesis-2000-021, 2000.

[11] U. Welander et al., "Absolute measures of image quality for the Sens-A-Ray direct digital intraoral radiography system," Oral Surg. Oral Med. Oral Pathol., vol. 80, pp. 345-50, 1995.

[12] International Commission on Radiation Units and Measurements, "Medical imaging-The assessment of image quality,", Rep. 54, 1996. 